PAPER • OPEN ACCESS

\title{
Diagrammatic approach to gas-liquid phase transition in statistical theory
}

To cite this article: Yu P Virchenko and L P Danilova 2019 J. Phys.: Conf. Ser. 1203012088

View the article online for updates and enhancements.

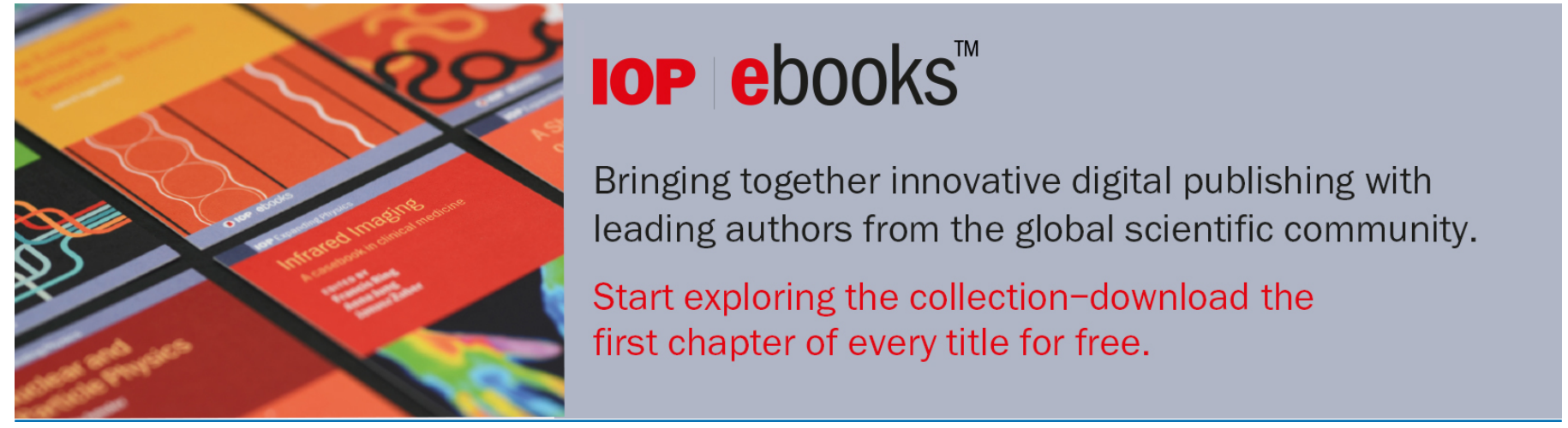

This content was downloaded from IP address 93.157 .144 .7 on $27 / 05 / 2020$ at $12: 28$ 


\title{
Diagrammatic approach to gas-liquid phase transition in statistical theory
}

\author{
Yu P Virchenko and L P Danilova \\ Department of Theoretical and Mathematical Physics, Belgorod State University, Belgorod, \\ Russia \\ E-mail: virch@bsu.edu.ru
}

\begin{abstract}
The lattice gas system is studied. In frameworks of statistical mechanics, it is proposed the analytic algorithm of approximations construction when pressure calculating. Algorithm is built on the basis of virial expansion using special small parameter $\nu$. The parameter is introduced by the prescription of the corresponding $\nu$-power weight to each Mayer diagram without contact vertexes. The obtained formulas describe the gas-liquid phase transition. The density dependence on pressure has the jump if the temperature is less than the critical one. The algorithm permits to calculate the phase diagrams contained the critical point. The formula of pressure obtained at null approximation corresponds to the self-consistent field approximation. Corrections to the critical temperature, the density jump value and the curves of phase diagrams are calculated in the approximation with the accuracy to first order on $\nu$. It is manifested the forbiddance of phase transition in one-dimensional system.
\end{abstract}

\section{Introduction}

Statistical theory of gas-liquid phase transition has the large history, but now there is not a mathematically substantiated method in frames of statistical mechanics for calculation of the thermodynamic characteristics of the transition even for such simple systems of statistical physics as one-atom gases. In this communication we point out the way of problem solving. It is proposed the algorithm for the phase $(P, T)$ - and $(\rho, T)$-diagrams calculation using the so-called lattice approximation in frames of the statistical mechanics formalism. These diagrams represents the first order phase transition lines which has the critical point. Besides, the algorithm permits to calculate the particle density $\rho$ as a function on the pressure $P$ if such a dependence has the jump. From the point of view of statistical mechanics formalism, phase transitions in lattice models are bifurcations of the probability distribution of a random Gibbs field on the lattice $\mathbf{Z}^{3}$ when the parameters of its probability distribution change. Bifurcations of Gibbs probability distributions are accompanied by the appearance of essential singularities in the analytical dependencies of expectations. Their presence greatly complicates the calculation of thermodynamic characteristics by analytical methods, since any method of their approximate calculation with guaranteed accuracy estimates implies that the availability of bifurcations should be manifested already in the lower orders of approximations.

As for the lattice gas models, it is possible to use an analytical approach to study the gasliquid phase transition. Such a possibility follows from the fact that the hysteresis is absent in such a transition despite the fact it is a phase transition of the first order one. It is quite sufficient to interpret the gas-liquid phase transition in the framework of the Lee and Yang theory 
of condensation [1]. In these works the concrete mathematical mechanism has been proposed according to which singularities corresponding the gas-liquid phase transition are appeared. In the Lee-Yang theory, the phase transition is associated with the presence of singularities of the variable $z$ which is named the fugacity. They lead to the division of the complex plane $z$ into two parts. Therefore, the pressure as a function of $z$ are represented by two analytical functions, which can not be connected with each other by the procedure of analytical continuation.

Main idea of our pressure approximations constructing dates back to the famous series of works [2]. It lies in the fact that if the correctly introduced radius $r_{0}$ of the interaction between particles tends to $\infty$, the values of thermodynamic functions should be equal to those that are found on the basis of the so-called approximation of the self-consistent field. In real systems, the interaction radius is not large compared to, for example, the average distance between the particles. However, this idea is important in ideological sense. It is due to the fact that the correlation radius is, indeed, very large in the parameter change domain where the phase transition occur. Thus, it plays the role of the interaction radius.

The presence of a first order phase transition in a lattice gas at sufficiently low temperatures is proved in the work [3]. It is done under conditions when the attraction region of the interaction potential plays a dominant role. It is also worth mentioning the work [4] performed at that time where similar results were obtained. This stage of statistical mathematical physics development is summarized in the monograph [5].

The problem under study in our communication analysed in [6] (see, also [7, 8]) where the Van-der-Waals limit for the lattice models that is in accordance with with the theme of our investigation. Result obtained in these works is analogous to zero approximation which is discussed in 5th section of our article.

\section{The lattice approximation}

We propose the solution of the problem basing on the lattice approximation. It permits to simplify essentially the solution of sufficiently routine problem that is named the exclusion of molecules volume. Such a procedure is necessary in order to take into account of strong repulsion of molecules at short distances. Let molecules may be occupy only points of the discrete set $\Lambda=\left\{\mathbf{x} \in \mathbf{R}^{3}: \mathbf{x}=a\left(n_{1} \mathbf{e}_{1}+n_{2} \mathbf{e}_{2}+n_{3} \mathbf{e}_{3}\right)\right\}$ which are contained in the cube $\Omega(\Lambda)$ with the side $a L$ where $a$ is lattice constant, $\mathbf{e}_{j}, j=1,2,3$ is a fixed orthogonal basis in $\mathbf{R}^{3}$ such that $L^{3}$ is the number of possible space positions of molecules, $|\Omega(\Lambda)|=(a L)^{3}$ is the system volume. Let us consider the lattice system of statistical mechanics (see, for example, [5]) with the hamiltonian

$$
\mathbf{H}[\rho]=-\mu \sum_{\mathbf{x} \in \Omega(\Lambda)} \rho(\mathbf{x})+\sum_{\{\mathbf{x}, \mathbf{y}\} \in \Omega(\Lambda)} U(\mathbf{x}-\mathbf{y}) \rho(\mathbf{x}) \rho(\mathbf{y})
$$

where $\mu$ is the chemical potential, $U(\mathbf{z})$ is the central symmetric interaction potential.

In order to account the strong molecules repulsion, we put $U(0)=\infty$. Here, $\rho(\mathbf{x})$ is an arbitrary dichotomous function defined on lattice sites $\Lambda$ which has values 0,1 . The interaction potential $U: \Lambda \mapsto \mathbf{R}$ is summable,

$$
\|U\| \equiv \sum_{0 \neq \mathbf{z} \in \Lambda}|U(\mathbf{z})|<\infty
$$

On the basis of the hamiltonian (1), it is introduced the Gibbs probability distribution $\mathrm{P}[\rho(\mathbf{x})]$ of the random dichotomous field with the space $\{\rho(\mathbf{x}) \in\{0,1\}: \mathbf{x} \in \Omega(\Lambda)\}$ of elementary events such that

$$
\mathrm{P}[\rho(\mathbf{x})]=\Xi^{-1} \exp (-\mathrm{H}[\rho] / T), T>0
$$


is the probability of the random realization $\rho(\mathbf{x}), T$ is the statistical temperature (the Botzmann constant is equal to unity),

$$
\Xi=\sum_{\{\rho(\mathbf{x})\}} \exp (-\mathrm{H}[\rho] / T)
$$

is the partition function. The expectation $\mathbf{E} \rho(\mathbf{x})$ represents the density $\rho$ of the lattice gas.

Dichotomous stationary random field with the space $\{\rho(\mathbf{x}) \in\{0,1\}: \mathbf{x} \in \Lambda\}$ of elementary events is defined by the expanding sequence of finite sets $\Omega(\Lambda)$ at $L \rightarrow \infty$. Such a limit passage is named the thermodynamic limit. It defines the probability distribution on the infinite probabilistic space by the limit $L \rightarrow \infty$ for each expectation. The probability distribution of infinite statistical system has the bifurcation when the parameter $T$ changing. Particularly, this bifurcation is manifested by the jump of the function $\rho(P)$ which represents the dependence the density $\rho$ on the pressure $P$. From the physical point of view. it is the phase transition of the first order

The pressure of lattice gas is defined on the basis of the partition function by the formula

$$
P=\lim _{L \rightarrow \infty} T \ln (\Xi /|\Omega(\Lambda)|) .
$$

To each function $\rho(\mathbf{x})$ we juxtapose the definite collection $X=\{\mathbf{x}: \rho(\mathbf{x})=1, \rho(\mathbf{x})=0, \mathbf{x} \notin X\}$, $n=\sum_{\mathbf{x} \in \Omega(\Lambda)} \rho(\mathbf{x})$ with mismatched components. It is defined with an accuracy to component permutations. Then, for any finite subset $X \subset \Omega(\Lambda)$, we have the value of the functional (Hamiltonian)

$$
H(X)=-|X|+\sum_{\{x, y\} \subset X} U(x-y)
$$

and the Gibbs probabilistic measure has the form

$$
\mathrm{P}[\rho(\mathbf{x})] \equiv \mathrm{P}\{X\}=\Xi^{-1} \exp (-H(X) / T) .
$$

Consequently, we have the following form of the partition function

$$
\Xi=\sum_{X \subset \Omega(\Lambda)} \exp (-H(X) / T)=\sum_{n=0}^{\infty} \frac{z^{n}}{n !} \sum_{X_{n} \in \Omega^{n}(\Lambda)} V_{n}\left(X_{n}\right),
$$

where $X_{n} \equiv X$, when $|X|=n$, and $V_{n}\left(X_{n}\right)=\exp \left(-\sum_{\{j, k\} \subset I_{n}} U\left(\mathbf{x}_{j}-\mathbf{x}_{k}\right) / T\right), I_{n}=\{1, \ldots, n\}$ are Boltzmann's functions. They are equal to zero when any pair of components in $X_{n}$-collection are coincide, $z=e^{\mu / T}$ is the fugacity parameter. There are the so-called group expansions of the pressure and the particle density on the $z$-powers (see, for example [9])

$$
P / T=\sum_{n=1}^{\infty} z^{n} b_{n}, \quad \rho=\sum_{n=0}^{\infty} z^{n+1}(n+1) b_{n+1} .
$$

They are differed from group expansions connected with continuous systems of statistical mechanics only such that integrals on particle locations are being replaced by summations on their discrete locations in sites of the cube lattice $\Lambda$, i.e.

$$
b_{n}=\frac{1}{n !} \sum_{X_{n-1} \in \Lambda^{n-1}} \sum_{G \in \mathcal{G}_{n}} \prod_{\{j, k\} \in G} W\left(\mathbf{x}_{j}-\mathbf{x}_{k}\right) .
$$

Here, the internal summation is done over all connected graphs $G$ from the class $\mathcal{G}_{n}$ with $n$ marked vertexes (Mayer's graphs). In formulas (4) each group coefficient $b_{n}(T)$ has the 
thermodynamic limit. It is connected with that the Ursell function $W(\mathbf{z})=e^{-U(\mathbf{z}) / T}-1$ is summable due to the summability of the potential.

Let the function $P(\rho, T)$ on $\rho$ and $T$ describes the pressure (3). It is represented in the form of series on density powers [9],

$$
P(\rho, T) / T=\rho\left(1-\rho \frac{d}{d \rho} \sum_{n=1}^{\infty} \frac{\rho^{n}}{n+1} \beta_{n+1}(T)\right)
$$

where coefficients $\beta_{n}(T), n \in \mathbf{N}$ are named the irreducible integrals. They are defined by the formula

$$
\beta_{n}(T)=\frac{1}{n !} \sum_{X_{n} \in \Lambda^{n}} \sum_{G \in \mathcal{F}_{n+1}} \prod_{\{j, k\} \in G} W\left(\mathbf{x}_{j}-\mathbf{x}_{k}\right), \quad n \in \mathbf{N} .
$$

This formula differs from the analogous one corresponding to continuous space of molecule locations such that integrations are replaced by summations. The internal summation is done over the class $\mathcal{F}_{n+1}$ of all connected graphs with $n+1$ vertexes which are not contact (see, for example, [10]). By the same way, it is fulfilled the analogous formula for the fugacity [9],

$$
z=\rho \exp \left(-\sum_{n=1}^{\infty} \beta_{n} \rho^{n}\right)
$$

\section{The algorithm construction of sequential approximations}

Building of approximations is based on the use of the expansion (5). It is necessary to determine approximations for each collection of coefficients $\beta_{n}(T), n \in \mathbf{N}$. They are built by the introducing a supplement "small" parameter $\nu$ into irreducible integrals. From physical point of view, the parameter $\nu$ corresponds to the ratio $r_{0} / r_{c}$ of the interaction radius to the correlation one. Since the strong repulsion of particles is not small, we define the new Urcell function

$$
W(\mathbf{z})=\widehat{W}\left(\mathbf{z}, r_{0}\right)-\delta_{\mathbf{z}, 0} .
$$

The particle volume is excluded in it. Let the function $\widehat{W}\left(\mathbf{z}, r_{0}\right)$ be small at $r_{0}^{-1} \ll 1$. Then irreducible integrals $\beta_{n}(T)$ are represented in the form

$$
\begin{gathered}
\beta_{n}(T)=\frac{1}{n !} \sum_{G \in \mathcal{F}_{n+1}} \sum_{G^{\prime} \subset G}(-1)^{\left|G^{\prime}\right|} \beta_{n}\left(G, G^{\prime}, r_{0}\right), \\
\beta_{n}\left(G, G^{\prime}, r_{0}\right)=\sum_{X_{n} \in \Lambda^{n}}\left(\prod_{\{j, k\} \in G^{\prime}} \delta_{\mathbf{x}_{j}, \mathbf{x}_{k}}\right)\left(\prod_{\{j, k\} \in G \backslash G^{\prime}} \widehat{W}\left(\mathbf{x}_{j}-\mathbf{x}_{k}, r_{0}\right)\right) .
\end{gathered}
$$

The internal summation in Eq.(6) is done over all subgraphs of the graph $G$. The particle volume exclusion in coefficients $\beta_{n}(T)$ consists of summations on the basis of Kroneker's symbols. Two kinds of edges appear in the graph $G$. Namely, the symbols $\delta_{\mathbf{x}_{j}, \mathbf{x}_{k}}$ correspond the edges of first kind, and the functions $\widehat{W}\left(\mathbf{x}_{j}-\mathbf{x}_{k}\right)$ correspond the edges of the second one. The graph $G^{\prime}$ is made up of the first kind edges. It consists of some subgraphs $G_{1}, G_{2}, G_{3}, \ldots, G^{\prime}=G_{1} \cup G_{2} \cup G_{3} \cup \ldots$ being not connected with each other. Then, despite that summations by Kronecker's symbols is simply fulfilled, in a result of such summations over all $X_{n}$, we obtain a complicated graph with multiple edges and contact vertexes. We name it as the derived graph. Thus, the summation on all derived graphs coresponding all graphs $G$ having $n$ vertexes should be fulfilled effectively in order to calculate irreducible integrals $\beta_{n}(T)$. It is true the following statement 
Theorem 1. There is the map $\mathrm{N}: \Sigma\left(G, G^{\prime}\right) \mapsto \mathbf{N}_{+}$such that for each pair $\left\langle G, G^{\prime}\right\rangle, G \in \mathcal{F}_{n+1}$, $G^{\prime} \subset G$ the following formula is fair for irreducible integrals

$$
\begin{aligned}
& \beta_{n}(T)=\frac{1}{n !} \sum_{G \in \mathcal{F}_{n+1}} \sum_{G^{\prime} \subset G}(-1)^{\left|G^{\prime}\right|} \times \\
& \times \sum_{X\left(\Sigma\left(G, G^{\prime}\right) \backslash\{1\}\right) \in \Lambda^{\left|\Sigma\left(G, G^{\prime}\right)\right|-1}} \prod_{\{j, k\} \subset \Sigma\left(G, G^{\prime}\right)} \widehat{W}^{\mathrm{N}(\{j, k\})}\left(\mathbf{x}_{j}-\mathbf{x}_{k}, r_{0}\right)
\end{aligned}
$$

where $\Sigma\left(G, G^{\prime}\right)$ is the vertex set of derived graph.

For each derived graph with the pair $\left\langle G, G^{\prime}\right\rangle$, we calculate the asymptotics of its contribution to corresponding irreducible integral $\beta_{n}(T)$ when $r_{0} \rightarrow \infty$. It is done in order to determine dependences on $\nu$ of irreducible integrals $\beta_{n}(T)$,

$$
\beta_{n}\left(G, G^{\prime}, r_{0}\right) \sim \frac{(-T)^{-\kappa\left(G, G^{\prime}\right)}}{\left(r_{0}^{3}\right)^{m\left(G, G^{\prime}\right)}} \int_{\left(\mathbf{R}^{3}\right)^{\left|\Sigma\left(G, G^{\prime}\right)\right|-1}} \prod_{\{j, k\} \subset \Sigma\left(G, G^{\prime}\right)} \widehat{U}^{\mathrm{N}(\{j, k\})}\left(\mathbf{x}_{j}-\mathbf{x}_{k}\right) d X\left(\Sigma\left(G, G^{\prime}\right) \backslash\{1\}\right)
$$

where $\kappa\left(G, G^{\prime}\right) \equiv \sum_{\{j, k\} \subset \Sigma\left(G, G^{\prime}\right)} \mathrm{N}(\{j, k\})$ is number of edges in derived graph with account of their multiplicity, $m\left(G, G^{\prime}\right)=1+\kappa\left(G, G^{\prime}\right)-\left|\Sigma\left(G, G^{\prime}\right)\right|$ is the characteristic indicator of derived graph. On the basis of this result, we prescribe the multiplier $\nu^{m\left(G, G^{\prime}\right)}$ to each term $\beta_{n}\left(G, G^{\prime}\right)$. So, irreducible integrals are some functions on $\nu$,

$$
\begin{aligned}
\beta_{n}(\nu, T) & =\frac{1}{n !} \sum_{G \in \mathcal{F}_{n+1}} \sum_{G^{\prime} \subset G}(-1)^{\left|G^{\prime}\right|} \nu^{m\left(G, G^{\prime}\right)} \beta_{n}\left(G, G^{\prime}\right), \quad \beta_{1}(\nu, T)=\beta_{1}, \\
\beta_{n}\left(G, G^{\prime}\right) & =\sum_{X\left(\Sigma\left(G, G^{\prime}\right) \backslash\left\{k_{1}\right\}\right) \in \Lambda^{\left|\Sigma\left(G, G^{\prime}\right)\right|-1}} \prod_{\{j, k\} \subset \Sigma\left(G, G^{\prime}\right)} \widehat{W}^{\mathrm{N}(\{j, k\})}\left(\mathbf{x}_{j}-\mathbf{x}_{k}\right) .
\end{aligned}
$$

Thus, we have defined the immersion of lattice gas model into more wide class of lattice models which is parametrized by the analytic $\nu$-dependence. It permits $t$ investigate the lattice gas quantitatively by means of building of asymptotic expansions over $\nu$. The original system is obtained at $\nu=1$.

\section{The location of phase transition in the space of thermodynamic parameters}

This section devotes two properties of lattice gas which are necessary for construction of $\nu$ expansions. They concern with the determination of phase transition point location. At first, it is necessary to define the relationship between $z$ and $\rho$ in this point. It is done on the basis of the equation

$$
\rho=z \exp \left(\sum_{n=1}^{\infty} \beta_{n}(T) \rho^{n}\right)
$$

According to the Lee-Yang theorem (see, [1],[5]), all zeroes of the partition function $\Xi$ being a polynomial on the fugacity $z$ are located on the circle with radius $\lambda^{-1}(T)$ if the interaction potential have no some negative values. Here,

$$
\lambda^{2}(T)=\exp \left[-\sum_{\mathbf{x} \in \Lambda} \widehat{U}(\mathbf{x}) / T\right] .
$$

Zeroes densely fill out the arc of the circle with the radius $\lambda^{-1}(T)$ when the thermodynamic limit is doing and the polynomial degree is tending to infinity. But the arc does not intersect the real axis. 
The phase transition is occur in the system when this arc occupies the circle completely, besides the point with real value. Consequently, the corresponding value $z$ where the phase transition may be realized is equal to $\lambda^{-1}(T)$ at $T<T_{c}$. Thus, when changing the parameter $T$, it is possible to implement the situation when, for some of its values, these analytical functions are a representation of a single analytical function, and, for others, the values of these functions are not analytical extensions of each other. Such a situation is demonstrated at the figure below. The location of zeros of the polynomial $\Xi$ on $z$ on the complex plane at $T>T_{c}$ and $T<T_{c}$ is performed at left(a) and right(b) parts of the figure, respectively, where $p(T)$ is the phase transition point.

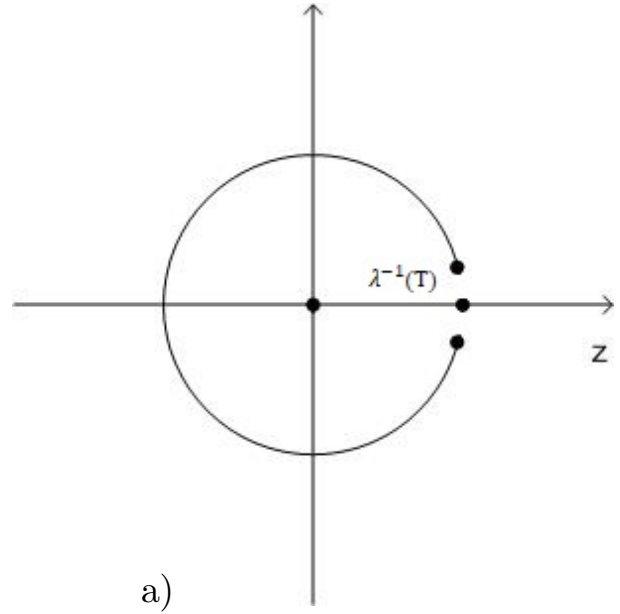

a)

Figure 1. The line of the concentration of partition function zeroes: a) $T>T_{c}$; b) $T<T_{c}$.

We have proved that such a situation takes place in the case when the potential $U$ is not attractive completely. The problem about the distribution of the partition function zeroes analysed in the work [11]. In this case, we cannot assert that zeroes are located on a circle, but they make up the point set that may adjoin to real axis only in the point $\lambda^{-1}(T)$.

Let

$$
p(z, T)=\frac{T}{|\Omega|} \ln \sum_{\rho(\mathbf{z})} z^{|\rho(\mathbf{z})|} \exp \left(-\frac{1}{T} \sum_{\{\mathbf{x}, \mathbf{y}\} \subset \Omega} U(\mathbf{x}-\mathbf{y}) \rho(\mathbf{x}) \rho(\mathbf{y})\right)
$$

be the pressure as the function on $z$ and $T$. Then the following assertion is true.

Theorem 2. Let the interaction potential $U$ in the Hamiltonian (1) defining the Gibbs random dichotomous field is summed and let the function $p(z, T)=T \ln \Xi /|\Omega(\Lambda)|$ is the pressure of the field. Then this function satisfies the functional equation

$$
p(z, T)=p\left(\left[\lambda^{2}(T) z\right]^{-1}, T\right)+T \ln z-\|\widehat{U}\| / 2
$$

for each $T>0$.

- The proof is done by the extraction of the multiplier $z^{|\Omega(\Lambda)|}$ out off the partition function.

In the case when the potential is not attractive completely, we may not assert that the split of the complex plane takes place, but, due to the relation

$$
\rho=z T^{-1} \frac{d}{d z} p(z, T)
$$

we obtain the following 
Theorem 3. The solution $\rho(z)$ of Eq.(7) satisfies the functional equation

$$
\rho(z)+\rho\left(\left[\lambda^{2}(T) z\right]^{-1}\right)=1 .
$$

Here, if the function $\rho(z)$ has some jumps, their lower and upper values are defined by left and right derivatives, correspondingly.

○ Eq.(8) is obtained by differentiating on $z$ of Eq. (7) both right and left at each point $z . \bullet$

From functional equation (8) one may conclude that if there is only one value $T$ at which the bifurcation of the probability distribution $\mathrm{P}[\rho]$ occurs, then the function $\rho(z)$ may be discontinuous only at $z=\lambda^{-1}(T)$. Firstly, it is connected with that the pressure $p(z, T)$ is a continuous, monotonically increasing function of $z$ for each fixed $T>0$ [5]. Then the presence of a phase transition, that is, the jump of density $\rho$ depending on $P$ corresponds to the presence of a fracture of the derived function $p(z, T)$ when $z$ changes and, accordingly, the presence of a jump in the function $\rho(z)$. Secondly, it is clear that at temperatures $T<T_{c}$ close enough to $T_{c}$ below, where $T_{c}$ is the critical temperature, there is only one pressure constancy interval $P$ from $\rho$ (the jump of the density depending on $\rho$ from $P$ ). The following statement, in a sense, extends applicability of the corollary of the Lee-Yang theorem on the value of the chemical potential $\mu$ corresponding the phase transition occurs for the potential $U$ which can take arbitrary values.

Theorem 4. If there are not some critical points in the temperature interval $\left(T_{c}, T_{*}\right)$ which are differed from $T_{c}$, the jump of the function $\rho(z)$ may be occured only at the value $z=\lambda^{-1}(T)$.

- From the equality (8) it follows that if at a given temperature the function $\rho(z)$ has a jump at a point $z$, then it also has a jump at point $\left[z \lambda^{2}(T)\right]^{-1}$. Due to the lack of critical points in the $\left(T_{c}, T_{*}\right)$, the jump $\rho(z)$ can only occur at one point $z$. From the condition of uniqueness of the jump, it should be $z=\left[z \lambda^{2}(T)\right]^{-1}$.

For the approximations constructing, it is important the following elementary proved statement which is a consequence of the symmetry between the full and empty lattice sites.

Theorem 5. At any temperature $T<T_{c}$, at which the system has only one jump of the function $\rho(P)$ which has the left and right extreme values $\rho_{-}(T), \rho_{+}(T)$, the density value $\rho=1 / 2$ always belongs to interval $\left[\rho_{-}(T), \rho_{+}(T)\right]$.

- The proof is done by replacing the summation variable. Namely, the function $\rho(\mathbf{x})$ is replaced by the equivalent dichotomous function $1-\rho(\mathbf{x})$ in the sum

$$
\rho=\mathbf{E} \rho(\mathbf{x})=\Xi^{-1} \sum_{\rho(\mathbf{z})} \rho(\mathbf{x}) \exp (-\mathrm{H}[\rho] / T) .
$$

After this, we obtain $\rho=1-\rho$, using periodic boundary conditions (see, [5]) for the system under consideration and also the value $z=\lambda^{-1}(T)$ if the phase transition occur. Hence, $\rho=1 / 2$ in such a situation.

Corrolary 1. The critical value $\rho_{c}$ of the density is equal $1 / 2$.

Corrolary 2. The value $p(T)$ of the pressure corresponding to the density jump is determined by the formula

$$
p(T)=P(1 / 2, T) .
$$

\section{Null approximation}

To calculate the pressure at null approximation $P^{(0)}(\rho, T)=\sum_{n=0}^{\infty} \beta_{n+1}(0) \rho^{n+1}$ according to the proposed procedure of approximations, it is necessary to find all pairs $\left\langle G, G^{\prime}\right\rangle$ which determine 
derived graphs with the characteristic indicator $m\left(G, G^{\prime}\right)=0$. It is proved that such pairs are $\langle G, \emptyset\rangle$ where $G$ is the graph with one edge of second kind and all pairs with $n \geq 2, G^{\prime}=G$, $G \in \mathcal{F}_{n+1}, n \in \mathbf{N}_{+}$. It is not complicated to prove the combinatorial formula which is valid for graphs with edges of first kind,

$$
\sum_{G \in \mathcal{F}_{n+1}}(-1)^{|G|}=-(n-1) !, \quad n \geq 1 .
$$

Taking into account the contribution into the coefficient $\beta_{1}(T)$ connected with the mentioned graph with second kind edge and summing all contributions of graphs with edges of first kind, we obtain

$$
T^{-1} P^{(0)}(\rho, T)=-\ln (1-\rho)-\frac{\rho^{2}}{2} \sum_{\mathbf{x} \in \Lambda}\left(e^{-\widehat{U}(\mathbf{x}) / T}-1\right) .
$$

This expression corresponds to the approximation of self-consistent field. In particular, the pressure has the following form for the model with the nearest-neighbours interaction

$$
\widehat{U}(\mathbf{z})=-U_{0} \text {, at }|\mathbf{z}|=a ; 0, \text { at }|\mathbf{z}| \neq a,
$$

$U_{0}>0, a>0$ is a lattice constant,

$$
T^{-1} P^{(0)}(\rho, T)=-\ln (1-\rho)-3 \rho^{2}\left(e^{U_{0} / T}-1\right) .
$$

The expression (11) has the bifurcation point $\left\langle\rho_{c}, T_{c}\right\rangle$ when the temperature $T$ is changing. At this point are fulfilled simultaneously two equalities $\partial P^{(0)} / \partial \rho=0$, $\partial^{2} P^{(0)} / \partial \rho^{2}=0$. Using these equations, we obtain that $\rho_{c}=1 / 2$ and the equation determining the temperature $T_{c}$,

$$
\sum_{\mathbf{x} \in \Lambda}(\exp (-\widehat{U}(\mathbf{x}) / T)-1)=4 .
$$

It is not complicated to prove the following statement.

Theorem 6. If the interaction potential $U$ has negative values at some points of the lattice $\Lambda$, so there is unique critical point $T_{c}>0$ that is the solution of Eq.(12).

In particular case, for the above-pointed model, the corresponding equation $4=6\left(e^{U_{0} / T}-1\right)$ has the explicit solution $T_{c}=U_{0}[\ln (5 / 3)]^{-1}$.

At zero approximation the phase $(P, T)$-diagram is found on the basis of Eq.(10),

$$
p(T)=T \ln 2-\frac{1}{8} T \sum_{\mathbf{x} \in \Lambda}\left(e^{-\widehat{U}(\mathbf{x}) / T}-1\right) .
$$

Theorem 7. The function $p(T)$ is concave and it is monotonically increase at the segment $\left[T_{*}, T_{c}\right]$ where $T_{*}$ is defined by the condition $p\left(T_{*}\right)=0$. The point $T_{*}$ is the unique solution of the equation

$$
8 \ln 2=\sum_{\mathbf{x} \in \Lambda}\left(e^{-\widehat{U}(\mathbf{x}) / T}-1\right) .
$$

Particularly, for the nearest neighbor model, we obtain

$$
p(T)=T \ln 2-\frac{3}{4} T\left(e^{-U_{0} / T}-1\right) .
$$

At last, the extreme points $\rho_{ \pm}(T)$ of the density jump that takes place at the pressure value $p(T)$ are determined as extreme solutions of the equation

$$
p(T)=P^{(0)}\left(\rho_{ \pm}(T), T\right),
$$


or that is equivalent

$$
-\ln \left(1-\rho_{ \pm}(T)\right)-\frac{1}{2}\left(\rho_{ \pm}^{2}(T)-\frac{1}{4}\right) \sum_{\mathbf{x} \in \Lambda}\left(e^{-\widehat{U}(\mathbf{x}) / T}-1\right)=\ln 2 .
$$

The function $\rho_{-}(T)$ is monotonically increasing over temperature and the function $\rho_{+}(T)$ is monotonically decreasing.

Asymptotic of the jump has been calculated explicitly at small temperature deviations $\Delta=T_{c}-T>0$ from the critical point,

$$
\rho_{+}(T)-\rho_{-}(T)=\alpha \frac{\sqrt{\Delta}}{T_{c}}, \alpha>0 .
$$

Thus, the jump has the critical exponent of the order parameter which is equal $1 / 2$. It is in agreement with the Landau thermodynamic theory of second order phase transitions.

\section{Correlation approximation}

Such an approximation corresponds to first order approximation on the parameter $\nu$,

$$
\left.P^{(1)} / T=-\rho^{2} \frac{d}{d \rho} \mathrm{Q}^{(1)}(\rho, T)\right), \quad \mathrm{Q}^{(1)}(\rho, T)=\sum_{n=2}^{\infty} \frac{\rho^{n}}{(n+1)} \beta_{n+1}^{(1)},
$$

since the pair correlation function does not vanish. For calculating of the coefficients

$$
\begin{gathered}
\beta_{n}^{(1)}=\frac{1}{n !} \sum_{G \in \mathcal{F}_{n+1}} \sum_{G^{\prime} \subset G: m\left(G, G^{\prime}\right)=1}(-1)^{\left|G^{\prime}\right|} \beta_{n}\left(G, G^{\prime}\right), \\
\beta_{n}\left(G, G^{\prime}\right)=\sum_{X\left(\Sigma\left(G, G^{\prime}\right) \backslash\{1\}\right) \in \Lambda^{\left|\Sigma\left(G, G^{\prime}\right)\right|-1}} \prod_{\{j, k\} \subset \Sigma\left(G, G^{\prime}\right)} \widehat{W}^{\mathrm{N}(\{j, k\})}\left(\mathbf{x}_{j}-\mathbf{x}_{k}\right),
\end{gathered}
$$

it is necessary to describe the class of pairs $\left\langle G, G^{\prime}\right\rangle, G \in \mathcal{F}_{n+1}$ which generate the derived graph with the characteristic indicator $m\left(G, G^{\prime}\right)=1$. It is proved that such graphs are represented in the rings form. Corresponding rings are made up of edges of second kind and their "effective vertexes" are some arbitrary graphs with edges of first kind which have no any contact vertexes.

In a result of solution of the combinatorial problem that consists the description of the class of such graphs, by the summation of contributions $\beta_{n}\left(G, G^{\prime}\right)$ of graphs with fixed number $n \geq 2$ of vertexes, one may find the coefficients

$$
\beta_{n}^{(1)}=\sum_{s=3}^{n+1} \sum_{m=2}^{s-1} \frac{(-1)^{s-m}(n+1)}{2(n+1-s) !}\left(\begin{array}{c}
s-1 \\
m
\end{array}\right) \frac{(n-m) !}{(s-m) !} \sum_{X_{m-1} \in \Lambda^{m-1}} \prod_{t=1}^{m}\left(e^{-\widehat{U}\left(\mathbf{x}_{t}-\mathbf{x}_{t+1}\right) / T}-1\right) .
$$

Series (11) with coefficients (14) is summable explicitly. In a result, the formula of the pressure is obtained with the accuracy of the first order if we take into account the expression $P^{(0)}(\rho, T)$

$$
\begin{gathered}
\frac{1}{T} P(\rho, T)=-\ln (1-\rho)-\frac{\rho^{2}}{4} \sum_{\mathbf{x} \in \Lambda}\left(e^{-\widehat{U}(\mathbf{x}) / T}-1\right)\left(3-e^{-\widehat{U}(\mathbf{x}) / T}\right)- \\
-\frac{a^{3}}{16 \pi^{3}} \rho^{2} \frac{d}{d \rho} \rho^{-1} \int_{\bar{\Lambda}} \ln (1-\rho(1-\rho)[\bar{W}(\mathbf{k})]) d \mathbf{k}, \bar{W}(\mathbf{k})=\sum_{\mathbf{x} \in \Lambda}\left(e^{-\widehat{U}(\mathbf{x}) / T}-1\right) e^{-i(\mathbf{k}, \mathbf{x})}
\end{gathered}
$$


is the sum of triple Fourier transformation of the Urcell function. The formula represents the periodic function on the space $\mathbf{R}^{3}$ of wave vectors $\mathbf{k}$. The function period is the so-called Brillouin zone $\bar{\Lambda}=[-\pi / a, \pi / a]^{3}$ of the reciprocal lattice of simple cubic lattice $\Lambda$.

On the basis of the obtained expression it has been calculated the correction of first order for the critical temperature

$$
\delta T^{-1}=-\frac{\nu}{2}\left[\sum_{\mathbf{x} \in \Lambda} \widehat{U}(\mathbf{x}) \exp \left(-\widehat{U}(\mathbf{x}) / T_{c}\right)\right]^{-1}\left[\sum_{\mathbf{x} \in \Lambda}\left(e^{-\widehat{U}(\mathbf{x}) / T}-1\right)^{2}+\frac{a^{3}}{\pi^{3}} \int_{\bar{\Lambda}} \frac{\bar{W}(\mathbf{k})(4 \bar{W}(\mathbf{k})-3)}{(4-\bar{W}(\mathbf{k}))^{2}} d \mathbf{k}\right]
$$

where $T=T_{c}$ in the Urcell function $\widehat{W}$ in the integrand expression and, therefore, $\bar{W}(0)=4$. Consequently, it is necessary to understand the integral in the main value.

In the same way, it is calculated the correction $\delta p(T)$ of the phase transition point $p(T)$

$$
\begin{gathered}
T^{-1} \delta p(T)=T^{-1} P^{(1)}(1 / 2, T)=\frac{1}{16} \sum_{\mathbf{x} \in \Lambda}\left(e^{-\widehat{U}(\mathbf{x}) / T}-1\right)^{2}-G_{1}(1 / 2), \\
G_{1}(1 / 2)=\frac{a^{3}}{16 \pi^{3}} \int_{\bar{\Lambda}} \ln (1-\bar{W}(\mathbf{k}) / 4) d \mathbf{k} .
\end{gathered}
$$

At last, corrections of the extreme points of the density jump are found with the same accuracy,

$$
\begin{gathered}
\delta \rho_{ \pm}(T)=\frac{1}{4} \frac{\rho_{ \pm}(T)\left(1-\rho_{ \pm}(T)\right)}{1-\left(1-\rho_{ \pm}(T)\right) \rho_{ \pm}(T) \bar{W}_{0}} \times \\
\times\left[\frac{a^{3}}{4 \pi^{3}} \int_{\bar{\Lambda}} \frac{\left(1-\rho_{ \pm}(T)\right)^{2} \bar{W}(\mathbf{k})}{1-\rho_{ \pm}(T)\left(1-\rho_{ \pm}(T)\right) \bar{W}(\mathbf{k})} d \mathbf{k}+\left(1-2 \rho_{ \pm}(T)\right) \sum_{\mathbf{x} \in \Lambda}\left(e^{-\widehat{U}(\mathbf{x}) / T}-1\right)^{2}\right] .
\end{gathered}
$$

\section{Conclusions}

The pressure $P(\rho, T)$ may be calculated with any accuracy by the $\nu$-expansion. It may be done by evaluation of functions $P^{(k)}(\rho, T)=-\rho^{2}\left(d Q^{(k)} / d \rho\right)$ with coefficients $\beta_{n}^{(k)}$ which are proportional to $\nu^{k}, k=2,3, \ldots$. The phase transition of first order is manifested by first two approximations. The expression in the integrand defining $P^{(1)}(\rho, T)$ exists only when the following conditions are fulfilled $\rho(1-\rho) W^{(m)}<1, W^{(m)}=\max _{\mathbf{k} \in \bar{\Lambda}}\{\bar{W}(\mathbf{k})\}$. Thus, the function $P^{(0)}(\rho, T)+P^{(1)}(\rho, T)$ has the natural domain

$$
\rho<\rho_{-}^{(m)} \equiv \frac{1}{2}\left(1-\left(1-4 / W^{(m)}\right)^{1 / 2}\right), \quad \rho>\rho_{+}^{(m)} \equiv \frac{1}{2}\left(1+\left(1-4 / W^{(m)}\right)^{1 / 2}\right) .
$$

Then the inverse function which is the dependence of $\rho$ on $P$ has the jump at this approximation by natural way.

Besides, if the formula (15) of the correction $\delta T$ is applied formally for the one-dimensional case, then $\delta T=\infty$. Thus, the approximation of the self-consistent field is not correct in onedimensional case. It is important since the application of the averaged field approximation predicts the phase transition for one-dimensional systems, but it is known the theorem stating that phase transitions are absent in one-dimensional systems with summable interaction potential [5]. 
IOP Conf. Series: Journal of Physics: Conf. Series 1203(2019) 012088 doi:10.1088/1742-6596/1203/1/012088

\section{References}

[1] Yang C N and Lee T D 1952 Statistical Theory of Equation of State and Phase Transitions. I.Theory of Condensation Phys. Rev. 87 404-409; II. Lattice Gas and Ising Model 410-419

[2] Kac M, Uhlenbeck G E and Hemmer P C 1963 On the van der Waals theory of the vapor-liquidequilibrium. I. Discussion of a onedimensional model J.Math. Phys. 4 216-228; II. Discussion of the distribution functions 229-247; III. Discussion of the critical region 5 60-74

[3] Ginibre J, Grossman A and Ruelle D 1966 Condensation of Lattice Gases Commun. Math. Phys. 3 187-193

[4] Fisher M E 1967 The Theory of Condensation and the Critical Point Physics. 3 255-283

[5] Ruelle D 1969 Statistical Mechanics. Rigorous Results (New York-Amsterdam: W.A. Benjamin, Inc.)

[6] Friedli S and Pfister C 2004 E Non-Analyticity and the van der Waals Limit J. Stat. Phys. 114 665-734

[7] Biskup M, Borgs C, Chayes J T and et al. 2004 Partition Function Zeros at First-Order Phase Transitions: Pirogov-Sinai Theory Journal of Statistical Physics 116, 97-155

[8] Pfister C E and van der Hofstad R 2005 On the Nature of Isotherms at First Order Phase Transitions for Classical Lattice Models Ensaios Matematicos. 9 1-90

[9] Isihara A 1971 Statistical physics (New York: Academic Press)

[10] Harary F 1969 Graph Theory (London: Addison-Wesley Publishing Company)

[11] Basuyev A G 2007 Hamiltonian, the phase boundaries and first order phase transitions. Generalization of the Lie-Yang theorem Theor. \& math. phys. 153 98-123 